\title{
LAS MEDIDAS DISCIPLINARIAS COMO PREDICTOR DE COMPORTAMIENTOS VIOLENTOS EN EL CENTRO ESCOLAR
}

\author{
Cristina Sánchez Romero \\ Universidad Nacional de Educación a Distancia \\ $M^{\text {a }}$ Carmen López Berlanga \\ Escuela Internacional de Doctorado (EIDUNED) \\ Isabel Martínez Sánchez \\ Universidad Nacional de Educación a Distancia \\ Rosalva Ruíz Ramírez \\ Universidad Autónoma de Sinaloa (México)
}

RESUMEN: Se presenta un estudio de la evolución de las faltas de disciplina en un período de tres años escolares para prevenir acciones violentas en el contexto educativo. Se han analizado 204 faltas cometidas por los estudiantes de primero a quinto de primaria desde un enfoque mixto de investigación. El análisis de correlación ( $\mathrm{Pr}>\mathrm{F}$ 0.037) es significativo con la variable que indica que los estudiantes del género masculino agreden mayormente a sus compañeros. Los resultados muestran que las principales faltas cometidas han sido faltas graves (50.5\%) como agredir a un compañero y faltar el respeto a un profesor. En conclusión, las infracciones disminuyen en la aplicación de las medidas disciplinarias en el contexto escolar.

PALABRAS CLAVE: Disciplina, violencia, contexto escolar, profesor.

\section{DISCIPLINARY MEASURES AS A PREDICTOR OF VIOLENT BEHAVIOR IN THE SCHOOL}

\begin{abstract}
A study of the evolution of the lack of discipline in a period of three school years is presented to prevent violent actions in the educational context. We analyzed 204 faults committed by students from first to fifth grade from a mixed method research approach. The correlation analysis ( $\mathrm{Pr}>$ F 0.037) is significant with the variable that indicates that students of the masculine gender attack mainly their peers. The results show that the main faults committed have been serious faults (50.5\%) such as assaulting a partner and disrespecting a teacher. In conclusion, the infractions decrease in the application of disciplinary measures in the school context.
\end{abstract}


KEYWORDS: School discipline, violence, school context, teacher.

Recibido: 21/12/2017

Aceptado: 14/05/2019

Correspondencia: Cristina Sánchez Romero, UNED, Facultad de Educación, C/ Juan del Rosal 14, 28040 Madrid. Email: csanchez@edu.uned.es.

\section{INTRODUCCIÓN}

Las formas y causas de la violencia en las aulas y en los centros se han analizado desde diferentes estudios y perspectivas (Olweus, 2001, 2006). Autores como Foshee et al. (2016) han analizado las diferencias entre las personas que intimidan pero no son víctimas de la intimidación y; los que son violentos y víctimas en los adolescentes, asociando a la victimización por intimidación a la ira, la depresión y la ansiedad para la perpetración de la violencia física. En otras investigaciones (Garaigordobil y Oñederra, 2010) se describen las relaciones existentes entre ser víctima de acoso escolar y ser agresor, se relaciona con la inteligencia emocional. Estos autores describen que tanto las víctimas como victimizadores tienen baja inteligencia emocional, baja responsabilidad y baja tolerancia a la frustración. Según estudios sobre el tipo de violencia que se producen dentro del ámbito educativo, y la permanencia de las actitudes tienen una prevalencia en diferentes organizaciones. En el informe Cisneros sobre Acoso y Violencia escolar en España (Piñuel y Oñate, 2006) se describen los comportamientos de acoso entre iguales (motes, insultos, acusaciones, mentiras, burlas, desprecios y exclusión)

Las faltas de disciplina, objeto de estudio de este artículo, que comenten los alumnos en el aula tienen un efecto en el desarrollo personal, social y educativo de los mismos y de sus compañeros y se encuentran dentro de las conductas que promueven situaciones de acoso y conflicto escolar. Los estudios sobre las conductas de intimidación en la escuela muestran otros factores influyentes en las mismas (Garaigordobil y Oñederra, 2010) como los niveles bajos de autoestima, motivación, inteligencia emocional y tolerancia a la frustración. Todo esto se une también a las conductas antisociales que algunos estudiantes presentan en el centro educativo. Autores como Marcus (2007) en la línea del modelo de agresión de Anderson y Bushman (2002) describe en la taxonomía de la influencia situacional de la agresión y el efecto en los adolescentes desde el estudio de las actitudes, entre otras, de provocación, frustración, y disconformidad. Detalla la importancia que tiene la agresión desde edades tempranas en la escuela infantil y su continuidad en distintos niveles educativos. Por ello, desde los diferentes ámbitos sociales y educativos se impulsan medidas e iniciativas para prevenir y erradicar la violencia en las escuelas, dotar a los alumnos de estrategias para afrontar; y resolver los conflictos escolares que, la mayoría de las veces, trascienden del ámbito escolar interfiriendo en el desarrollo psicosocial de los adolescentes (tanto actores, como víctimas, como meros observadores), ahondando en la idea que ya han defendido muchos investigadores y expertos 
sobre este tema; y que establece una relación directa entre la prevención de la violencia y la mejora de la convivencia. (The Anti-Bullying Alliance, 2009; Van Goethem, Scholte, y Wiers, 2010)

Ante esta situación problemática debemos reflexionar desde la Comunidad Educativa y todos los agentes implicados sobre la disciplina en el centro y aula.

La falta de disciplina es uno de los objetivos de este artículo y de la actuación del profesorado en el aula, además de otros programas (Evans, Fraser y Cotter, 2014) como el plan de convivencia que se desarrolla en los centros educativos con la finalidad de desarrollar un seguimiento de los comportamientos y actitudes nocivas entre los estudiantes y dar respuesta e implicación por parte del profesorado a las conductas disruptivas (Yoon y Kerber, 2003)

\section{La indisciplina como indicador de violencia en las aulas}

En el aula educativa, los indicadores de violencia pueden ser percibidos tanto por el profesorado como por los estudiantes. La falta de indisciplina se convierte en un predictor del conflicto.

En este sentido, hemos analizado estudios en diferentes niveles educativos que muestran como factor común la falta de disciplina, por un lado, dirigida al profesorado (Idu y Ojedapo, 2011; Maphosa y Mammen, 2011) y, por otro, la relación de la falta de disciplina con los niveles de agresión en las escuelas (Watson, 2005)

El docente incide sobre los comportamientos que influyen y perturban el clima del aula no reconocidos por los estudiantes implicados (Hastie y Siedentop, 2006) y, también sobre el grado de perturbación en el aula y; la actitud del profesorado para evitar estas conductas (Garaigordobil y Oñederra, 2010)

La calidad del aula y el manejo efectivo de la misma por los maestros estudiada por Rimm-Kaufman, Nathanson, y Brock (2009) se vincula con un mayor autocontrol comportamental y cognitivo de los niños. Por lo que la asunción de las normas y disciplina del aula evitaría un clima disruptivo.

En esta situación, se ha de tener en cuenta otros factores externos como las conductas docentes negativas, (Brophy-Herb, Lee, Nievar, y Stollak, 2007) la violencia en la familia (McKinney, Caetano, Ramisetty-Mikler y Nelson, 2009) y el contexto social (Chang, 2004) dónde se asimilan precozmente.

El desarrollo de prácticas disciplinarias con resultado positivo para el centro y el aula han sido analizadas desde diferentes perspectivas. Por ejemplo, Preston (1991) incluye el uso de procedimientos disciplinarios como intervención y apoyo de comportamiento positivo y, justicia restaurativa como formas efectivas en el centro. Otros autores como Hoffman (1994) describe la teoría de la internalización y las respuestas afectivas; y cognitivas de los niños en encuentros disciplinarios. Propone los ejercicios disciplinarios desde la capacidad de empatía de los niños y de sus sentimientos.

En el sentido de la relación entre la disciplina y rendimiento, autores como Kinsler (2013) estudió los efectos indirectos del comportamiento disruptivo. La disciplina 
tiene una influencia positiva general en el rendimiento de los estudiantes. También describe que la variación entre escuelas en las políticas de disciplina se relaciona con la maximización de logros.

El papel del profesor es esencial en este sentido aunque también debemos analizar la ética en sus decisiones en la disciplina y las sanciones (Meirieu, 2001) y la gestión democrática de las normas haciendo frente a la disrupción desde el diálogo educativo del contexto (Torrego Seijo y Aguado, 2006). El orden y la disciplina son herramientas claves en la escuela para la prevención de comportamientos inadecuados (Van der Horst y Narodowski, 1999).

La falta de disciplina también influye en el impacto emocional del docente (Galand, Lecocq y Philippot, 2007) y repercute en su bienestar emocional, por ello, requiere del apoyo de otros agentes educativos del centro.

Las relaciones entre el profesor y el estudiante es un factor analizado también por Chen y Avi Astor (2010) para la prevención de violencia en las aulas. En este sentido, para prevenir un comportamiento inapropiado que puede afectar a la escuela, se requiere la participación de los padres y de la comunidad (McKinney et al., 2009; Peterson y Skiba, 2001). El entorno social y físico de la escuela ofrece oportunidades de intervención para reducir la violencia escolar (Johnson, 2009)

\section{Las medidas disciplinarias: Plan de convivencia del centro educativo}

La generación de programas de convivencia en los centros educativos es una medida necesaria que garantizará el cumplimento de las normas en el aula y en el centro.

Los programas de prevención han sido siempre una medida de erradicación de la violencia en las aulas y en los centros. Existen numerosas investigaciones nacionales e internacionales que han dado lugar a proyectos significativos sobre las medidas educativas para evitar los conflictos en el aula y comportamientos inapropiados. Destacan algunos como el Piscataway Project de Hunter, Elias y Norris (2001) basado en la escuela que se centra en los enfoques instructivos de prevención de la violencia. El proyecto Responding in Peaceful and Positive Ways Project (RIPP) de Farrell, Meyer, y White (2001) que evalúa las respuestas pacíficas y positivas (RIPP) para reducir la violencia desde la violaciones disciplinarias en los participantes del proyecto. En el programa universal de violencia basado en la escuela primaria PeaceBuilders (Flannery et al., 2003) se analiza la mejora de la competencia social infantil como herramienta que reduce el comportamiento agresivo en la escuela. En el programa Universal school-based violence prevention program on social-cognitive (Simon et al., 2008) se analizan los factores sociocognitivos asociados con la agresión y el comportamiento violento en la adolescencia temprana. También el Peacemakers Program (Shapiro, Burgoon, Welker, y Clough, 2002) se describe un proyecto de prevención de la violencia desde el componente de la prevención primaria en la escuela. 
En este sentido, destacamos en nuestro país un modelo estratégico para la actuación global sobre conflictos de convivencia en centros educativos: "Proyecto Atlántida" (Luengo, 2006) para la convivencia y disciplina en los centros escolares sobre aspectos como "Obtener un conocimiento más ajustado del alumnado que permita una mejor respuesta educativa".

Aunque se requiere seguir investigando en este ámbito para encontrar una mejor implicación de los desarrolladores de programas y una mayor investigación e implementación de los mismos como describen Schultes, Stefanek, van de Schoot, Strohmeier, y Spiel (2014) en el análisis del programa School-based Violence Prevention Program ViSC.

Por ello, sentimos la necesidad de analizar los comportamientos violentos a través de las normas de conducta y medidas disciplinarias que garanticen el cumplimiento de la convivencia. Para ello, hemos seleccionado aquellas normas (Tabla $1 ; 2 ; 3$ ) que al menos han de ser obligatorias para analizar las posibles sanciones y medidas disciplinarias en los alumnos del Decreto 15/2007, de 19 de abril

Tabla 1. Normas de Conducta. Art.3. Decreto 15/2007, de 19 de abril

\section{Artículo 3.- Las Normas de Conducta.}

a) La asistencia a clase.

b) La puntualidad a todos los actos programados por el centro.

c) El mantenimiento de una actitud correcta en clase, no permitiéndose el uso de móviles, otros dispositivos electrónicos o cualquier objeto que pueda distraer al propio alumno o a sus compañeros.

d) El respeto a la autoridad del Profesor, tanto dentro de la clase como en el resto del recinto escolar.

e) El trato correcto hacia los compañeros, no permitiéndose, en ningún caso, el ejercicio de violencia física o verbal.

f) La realización de los trabajos que los Profesores manden realizar fuera de las horas de clase.

g) El cuidado y respeto de todos los materiales que el centro pone a disposición de alumnos y Profesores.

h) El cuidado de las instalaciones y del conjunto del edificio escolar.

Para el análisis de las sanciones ante el incumplimiento de las normas de conducta se han tenido en cuenta, del anterior Decreto, el artículo "Artículo 17.- Criterios para la adopción de sanciones" y "Artículo 18.- Circunstancias atenuantes y agravantes" que se han de adecuar a las edades de los alumnos y sus situaciones personales y sociales. 
Tabla 2. Criterios de sanciones. Decreto 15/2007, de 19 de abril

"Artículo 17.- Criterios para la adopción de "Artículo 18.- Circunstancias atenuantes y
sanciones".
agravantes"

Criterios a considerar en la adopción de 1. Para la graduación de las sanciones se sanciones disciplinarias y de medidas pro- apreciarán las circunstancias atenuantes o visionales: agravantes que concurran en el incumplia) La imposición de sanciones tendrá fina- miento de las normas de conducta.

lidad y carácter educativo, y procurará la 2. Se considerarán circunstancias atenuanmejora de la convivencia en el centro. tes:

b) Se deberán tener en cuenta, con carácter a) El arrepentimiento espontáneo.

prioritario, los derechos de la mayoría de b) La ausencia de intencionalidad.

los miembros de la comunidad educativa y c) La reparación inmediata del daño caulos de las víctimas de actos antisociales, de sado.

agresiones o de acoso. 3 3. Se considerarán circunstancias agravan-

c) No se podrá privar a ningún alumno de tes:

su derecho a la educación obligatoria. $\quad$ a) La premeditación y la reiteración.

d) No se podrán imponer correcciones con- b) El uso de la violencia, de actitudes ametrarias a la integridad física y la dignidad nazadoras, desafiantes o irrespetuosas, de personal del alumno. menosprecio continuado y de acoso dentro e) Se valorarán la edad, situación y circuns- o fuera del centro.

tancias personales, familiares y sociales c) Causar daño, injuria u ofensa a compadel alumno, y demás factores que pudieran ñeros de menor edad o recién incorporados haber incidido en la aparición de las con- al centro.

ductas o actos contrarios a las normas es- d) Las conductas que atenten contra el detablecidas.

recho a no ser discriminado por razón de

f) Se deberán tener en cuenta las secuelas nacimiento, raza, sexo, convicciones popsicológicas y sociales de los agredidos, así líticas, morales o religiosas, así como por como la alarma o repercusión social creada padecer discapacidad física o psíquica, o por las conductas sancionables. por cualquier otra condición personal o cirg) Las sanciones deberán ser proporciona- cunstancia social.

les a la naturaleza y gravedad de las faltas e) Los actos realizados en grupo que atencometidas, y deberán contribuir a la mejora ten contra los derechos de cualquier miemdel clima de convivencia del centro. bro de la comunidad educativa.

A continuación, destacamos la clasificación de las faltas de disciplina, referida a aquellas conductas que infrinjan las normas de convivencia del centro. La clasificación de las mismas: son leves, graves y muy graves que tienen que estar en el Reglamento de Régimen Interior del Centro y en lo dispuesto en el Decreto anteriormente citado. 


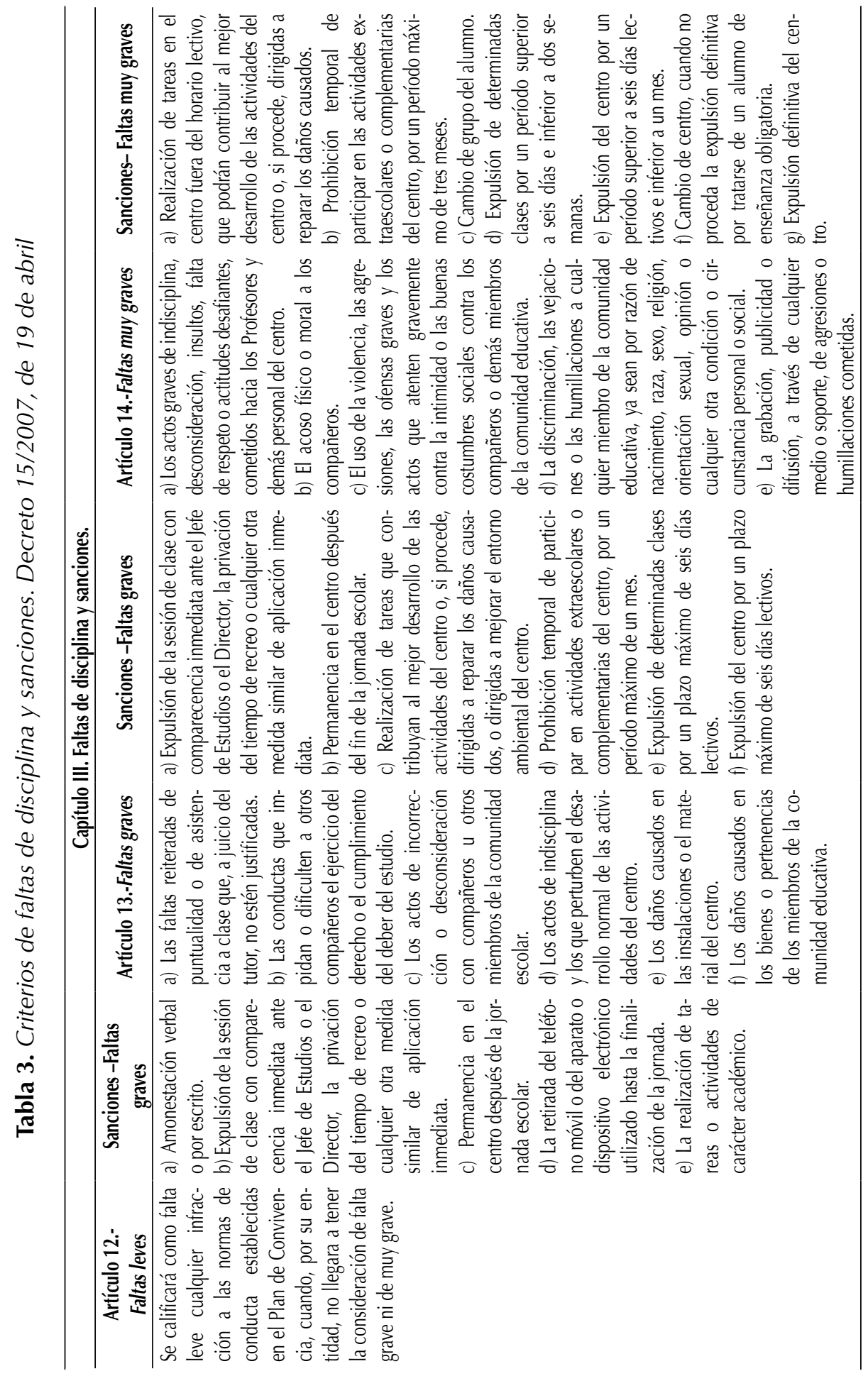


CRISTINA SÁNCHEZ - Mª CARMEN LÓPEZ - ISABEL MARTÍNEZ - ROSALVA RUÍZ

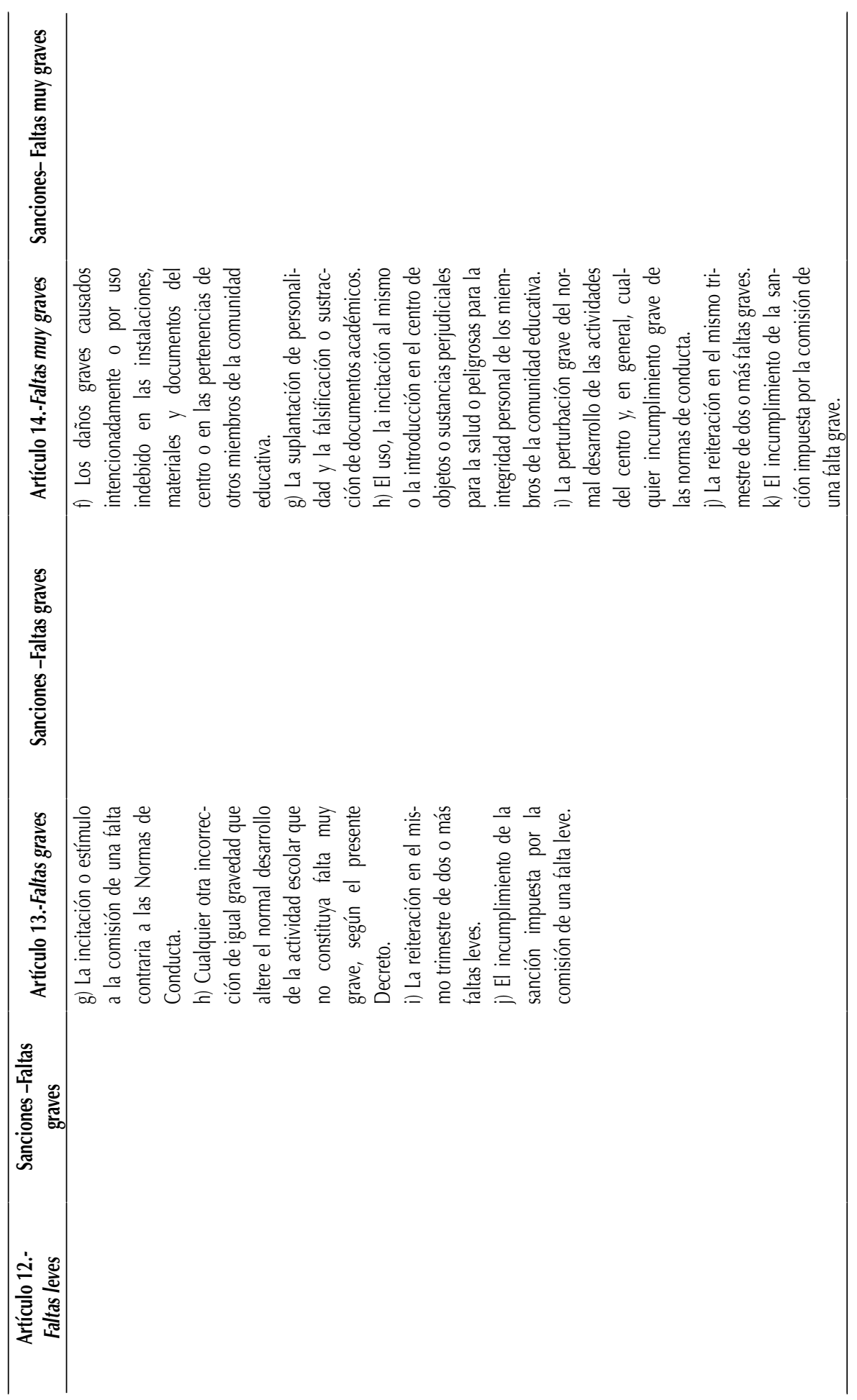




\section{Método}

Este artículo muestra el análisis de las diferentes infracciones disciplinarias (leves, graves y muy graves) que realiza los estudiantes durante un año escolar en el aula y/o centro educativo. En total se han analizado 204 faltas de disciplina durante el período escolar 2013-2016. El enfoque metodológico del estudio se desarrolló desde el paradigma de investigación mixto en la línea de autores como Creswell y Clark, (2010a); Denzin (2010); Johnson, Onwuegbuzie, y Turner (2007); Teddlie y Tashakkori (2010); Teddlie y Yu (2007); Terrell (2012a).

El objetivo general de la investigación es analizar la falta de disciplina que los escolares realizan durante el curso académico. Entre los objetivos específicos destacamos:

- Identificar el número de faltas de disciplina que se desarrollan en un centro educativo.

- Analizar el grado de gravedad de la indisciplina cometida (leve, grave, muy grave)

- Detectar el motivo que produce que se desarrolle la indisciplina en el aula y/o centro.

- Establecer la sanción pertinente para la corrección de la falta cometida.

Atendiendo a la normativa general del Decreto 15/2007, y a la normativa en particular del Régimen Interior de Centro seleccionado hemos analizado la falta de disciplina. Para categorizar las faltas de disciplina se han utilizado las siguientes variables: año escolar, curso académico, género, tipo de falta (leve, grave, muy grave) y el motivo. Para ello, se diseñó una matriz de datos en base a las fichas del estudiante que desarrolla el profesor sobre la falta de disciplina con los campos formados por las variables citadas anteriormente.

\section{MUeSTRA}

La muestra participante compuesta por 300 estudiantes de primaria de diferentes cursos académicos. De esta muestra seleccionada no probabilística se analizaron las diferentes faltas cometidas por los mismos, en total 204 faltas de los cuales el $(76 \%)$ pertenecen a faltas cometidas por el género masculino y el (24\%) pertenecen a las faltas cometidas por el género femenino, caracterizadas por su tipología (Leve, Grave y Muy grave)

\section{Análisis de los resultados}

En el año escolar 2013-2014, 49\% de la muestra estudiada han cometido faltas de conductas disruptivas, $24.5 \%$ en $2014-2015$ y $26.5 \%$ en $2015-2016$.

El total de las faltas cometidas fueron 204, los chicos fueron quienes más faltas cometieron (76\%), mientras que las chicas realizaron $24 \%$ de las faltas. La tipología de las faltas cometidas son leves, graves y muy graves. 
La Tabla 4 muestra que por cursos, el $15.7 \%$ de las alumnas y los alumnos de primer grado han cometido actos de indisciplina, $6.4 \%$ segundo, $10.3 \%$ tercero, $14.2 \%$ cuarto y $48.5 \%$ de quinto.

El curso escolar que mayor porcentaje de faltas cometidas fue el curso escolar de quinto con $48.5 \%$, el de menor porcentaje fue el grado de segundo con $6.4 \%$. Además, el género no fue restrictivo, ya que ambos cometieron faltas.

Tabla 4. Actos de disciplina por género y curso académico

\begin{tabular}{|l|c|c|c|}
\hline \multirow{2}{*}{ Curso escolar } & \multicolumn{2}{|c|}{ Género } & \multirow{2}{*}{$\begin{array}{c}\text { Total } \\
\mathbf{( \% )}\end{array}$} \\
\cline { 2 - 3 } & Masculino (\%) & Femenino (\%) & 15.7 \\
\hline Primero & 12.3 & 3.4 & 6.4 \\
\hline Segundo & 5.4 & 1 & 10.3 \\
\hline Tercero & 6.4 & 3.9 & 14.2 \\
\hline Cuarto & 11.8 & 2.5 & 48.5 \\
\hline Quinto & 37.3 & 11.3 & 95,3 \\
\hline Total & 73,2 & 22,1 & \\
\hline
\end{tabular}

La Tabla 5 muestra el nivel de faltas que se cometieron, mayormente fueron graves (50.5\%), 37.3\% de los alumnos y $13.2 \%$ de las alumnas; posteriormente leves $(27 \%)$ y por último, faltas muy graves $(22.5 \%)$.

Tabla 5. Faltas cometidas por género

\begin{tabular}{|l|c|c|c|}
\hline \multicolumn{1}{|c|}{ Faltas: } & Masculino (\%) & Femenino (\%) & Total (\%) \\
\hline Leve & 21.1 & 5.9 & 27 \\
\hline Grave & 37.3 & 13.2 & 50.5 \\
\hline Muy grave & 17.6 & 4.9 & 22.5 \\
\hline
\end{tabular}

En la Tabla 6, se muestra que el alumnado del año escolar 2013-2014, fueron quienes más faltas de conductas disruptivas han cometido con 49\%. 26.5\% los del año escolar 2015-2016 y 24.5\% los del ciclo 2014-2015. Este dato indica que entre más reciente es el año escolar el nivel de faltas ha disminuido, lo que probablemente se traduzca en una mayor sensibilización del tema, y por ende en un cambio en la conducta, disminuyendo la indisciplina. Sin embargo, los datos muestran que las faltas de indisciplina se cometieron en todos los ciclos escolares, sobresaliendo las faltas graves.

Tabla 6. Faltas cometidas por año escolar

\begin{tabular}{|l|c|c|c|c|}
\hline \multicolumn{1}{|c|}{ Año escolar } & Falta leve $\%$ & Falta grave $\%$ & $\begin{array}{c}\text { Falta muy } \\
\text { grave } \%\end{array}$ & Total $\%$ \\
\hline $2013-2014$ & 13.7 & 23 & 12.3 & 49 \\
\hline $2014-2015$ & 4.4 & 12.7 & 7.4 & 24.5 \\
\hline $2015-2016$ & 8.8 & 14.7 & 2.9 & 26.5 \\
\hline Total & 26.9 & 50.4 & 22.6 & \\
\hline
\end{tabular}


El principal motivo de falta que se repite (Tabla 7) es la agresión a los/as compañeros/as, la cual es catalogada como una manifestación física, posteriormente la falta de respeto, la conducta desafiante y las amenazas, las cuales, se clasifican como manifestaciones psicológicas.

Tabla 7. Relación entre el año académico y motivo del tipo de falta

\begin{tabular}{|l|c|c|c|c|c|}
\hline \multirow{2}{*}{ Ciclo escolar } & \multicolumn{3}{|c|}{ Motivo } & \multirow{2}{*}{ Total } \\
\cline { 2 - 5 } & Agresión & Falta de respeto & Conducta desafiante & Amenazas & \\
\hline $2013-2014$ & 33 & 20 & 16 & 5 & 74 \\
\hline $2014-2015$ & 13 & 17 & 8 & 0 & 38 \\
\hline $2015-2016$ & 26 & 17 & 4 & 0 & 47 \\
\hline Total & 72 & 54 & 28 & 5 & 159 \\
\hline
\end{tabular}

Las agresiones que cometieron los hombres casi cuadruplican las faltas en comparación con las mujeres, los mayores motivos son las agresiones y las conducta desafiantes (Tabla 8). El mayor acto de indisciplina que comenten las mujeres obtienen son, al igual que los hombres las agresiones.

En definitiva, y con base en las diferentes tablas comparativas, el mayor número de faltas que se cometieron son graves, destacando las agresiones, la falta de respeto y la conducta desafiante. El perfil de los amonestados es el de un chico de los últimos cursos, $5^{\circ}$ o $6^{\circ}$ de primaria.

Tabla 8. Relación entre género y motivo

\begin{tabular}{|l|c|c|c|c|c|}
\hline \multirow{2}{*}{ Ciclo escolar } & \multicolumn{3}{|c|}{ Motivo } & \multirow{2}{*}{ Total } \\
\cline { 2 - 5 } & Agresión & Conducta desafiante & Amenazas & Falta de respeto & \\
\hline Hombres & 53 & 46 & 22 & 5 & 126 \\
\hline Mujeres & 19 & 8 & 6 & 0 & 33 \\
\hline Total & 72 & 54 & 28 & 5 & 159 \\
\hline
\end{tabular}

\section{Análisis de correlación}

La Tabla 9 muestra que el análisis de correlación es significativo para la variable que indica que los estudiantes del género masculino agreden mayormente a sus compañeros, en contraste con las mujeres.

El alumnado que cursa el año escolar 2015-2016 les faltan más el respeto al profesor en comparación que los del año escolar 2014-2015 y 2013-2014. Existe diferencia significativa en que las alumnas y los alumnos que han cometido faltas leves son quienes interrumpen las clases constantemente, tienen mal comportamiento reiterado y no traen la tarea constantemente.

Asimismo, existe diferencia significativa en que a menor año escolar, es mayor el mal comportamiento reiterado. 
Tabla 9. Correlaciones entre variables

\begin{tabular}{lccc}
\hline \multicolumn{1}{c}{ Variables } & Rho & Pr $>$ F & Significancia \\
\hline Año escolar - Falta de respeto al profesor/a & 0.200 & 0.004 & ${ }^{*}$ \\
\hline Interrumpir las clases - Faltas de disciplina & -0.279 & 0.000 & ${ }^{*}$ \\
\hline Género - Agredir a sus compañeros/as & -0.146 & 0.037 & ${ }^{*}$ \\
\hline Mal comportamiento reiterado - Faltas de disciplina & -0.168 & 0.016 & ${ }^{*}$ \\
\hline Mal comportamiento reiterado - Año escolar & -0.159 & 0.023 & ${ }^{*}$ \\
\hline Faltas de disciplina - No traer la tarea constantemente & -0.142 & 0.043 & ${ }^{*}$ \\
\hline
\end{tabular}

*Significativo, **Altamente significativo

En la Figura 1 se muestra la relación que existente entre las unidades hermenéuticas analizadas de las principales faltas cometidas por las y los estudiantes.

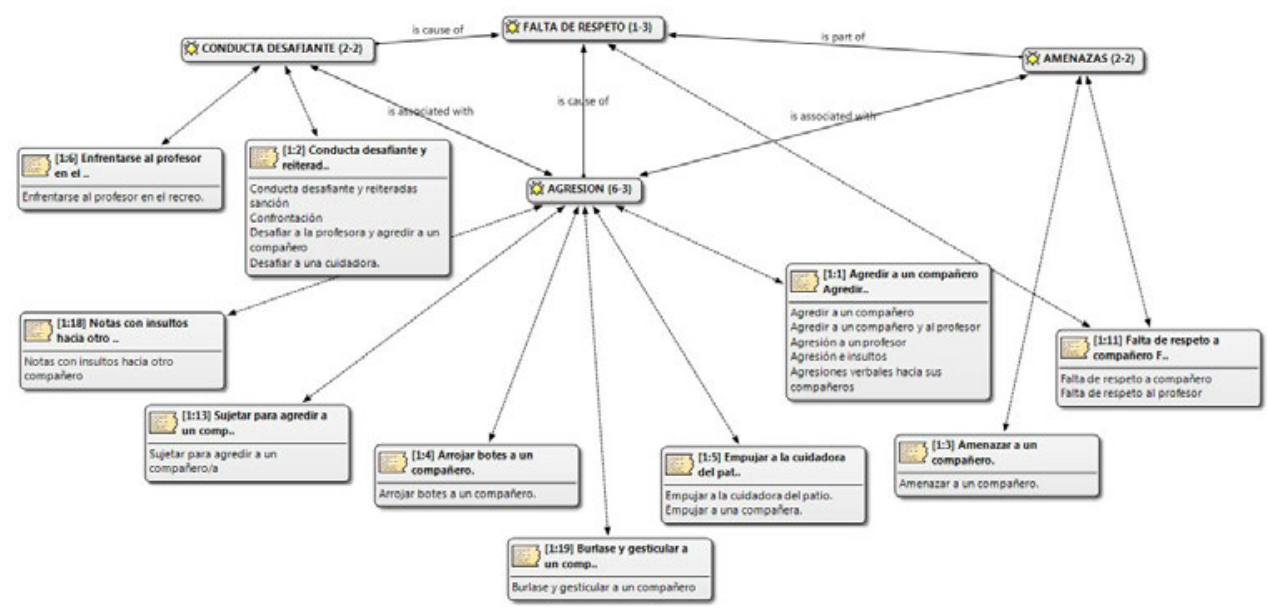

Figura 1. Falta de disciplina (Nodo: Agresión, Amenazas, Conducta desafiante, Falta de Respeto)

La agresión es una de las conductas predominantes que necesitan de medidas disciplinarias, para su corrección desde edades tempranas. Asimismo, la agresión es causa de la falta de respeto tanto al profesorado como a otros/as estudiantes.

Entre las unidades hermenéuticas analizadas se comprueba que el nodo (amenaza) está, asociado con el nodo (agresión) y, a su vez, al nodo (falta de respeto).

\section{Motivo de indisciplina, tipo de falta y sanción adoptada}

A continuación, en la Tabla 10, se analiza la actitud disruptiva, tipo de falta y la sanción que se adopta desde el centro como medida de mejora de la conducta. 
Tabla 10. Motivos de indisciplina, faltas y sanciones

\begin{tabular}{|c|c|c|c|}
\hline & Motivo de indisciplina & Tipo Falta & Sanciones adoptadas en el centro \\
\hline \multirow{7}{*}{ 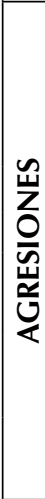 } & Agredir a un compañero/a & MG & $\begin{array}{l}\text { Permanecerá en el centro después del horario } \\
\text { lectivo realizando tareas. }\end{array}$ \\
\hline & $\begin{array}{l}\text { Agredir a un compañero/a } \\
\text { y al profesor/a }\end{array}$ & MG & Expulsión del centro 6 días a un mes. \\
\hline & Agresión a un profesor/a & MG & Expulsión de 6 días. \\
\hline & Agresión e insultos & MG & Expulsión del centro 6 días. \\
\hline & $\begin{array}{l}\text { Agresiones verbales hacia } \\
\text { sus compañeros/as }\end{array}$ & G & Expulsión del centro 3 días. \\
\hline & $\begin{array}{l}\text { Sujetar para agredir a un } \\
\text { compañero/a }\end{array}$ & MG & Expulsión del centro 6 días a un mes. \\
\hline & $\begin{array}{l}\text { Alentar el mal comporta- } \\
\text { miento }\end{array}$ & G & $\begin{array}{l}\text { Cambio de clase temporal. } \\
\text { Amonestación verbal o por escrito. }\end{array}$ \\
\hline \multirow{3}{*}{ 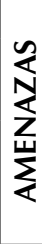 } & Confrontación & G & Amonestación verbal o por escrito. \\
\hline & $\begin{array}{l}\text { Amenazar a un } \\
\text { compañero/a }\end{array}$ & G & Expulsión de 3 días. \\
\hline & $\begin{array}{l}\text { Incitar el enfrentamiento } \\
\text { entre compañero }\end{array}$ & G & $\begin{array}{l}\text { Expulsión del recreo durante } 15 \text { días donde se } \\
\text { realizarán tareas con el orientador para corregir } \\
\text { la conducta. }\end{array}$ \\
\hline \multirow{5}{*}{ 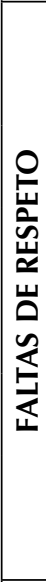 } & $\begin{array}{l}\text { Insultar a sus compañeros/ } \\
\text { as }\end{array}$ & G & $\begin{array}{l}\text { Pedir perdón, escribir una disculpa. } \\
\text { No salir en el recreo durante una semana y en ese } \\
\text { tiempo realizará tareas, trabajando la empatía. }\end{array}$ \\
\hline & $\begin{array}{l}\text { Interrumpir la clase cons- } \\
\text { tantemente }\end{array}$ & G & $\begin{array}{l}\text { Cambio de clase a nivel inferior o superior du- } \\
\text { rante } 15 \text { días. }\end{array}$ \\
\hline & $\begin{array}{l}\text { Lanzar un objeto al } \\
\text { profesor/a }\end{array}$ & MG & $\begin{array}{l}\text { Expulsión de la sesión de clase con comparecencia } \\
\text { inmediata ante el Jefe de Estudios o el Director, la } \\
\text { privación del tiempo de recreo o cualquier otra me- } \\
\text { dida similar de aplicación inmediata. }\end{array}$ \\
\hline & $\begin{array}{l}\text { Falta de respeto al } \\
\text { profesor/a }\end{array}$ & MG & Expulsión de 3 días. Reflexión de su conducta. \\
\hline & $\begin{array}{l}\text { Falta de respeto a } \\
\text { compañero/a }\end{array}$ & MG & $\begin{array}{l}\text { Expulsión del recreo durante } 15 \text { días donde se reali- } \\
\text { zaran tareas para mejorar la conducta (se trabajará la } \\
\text { empatía, las consecuencias, figura del mediador). }\end{array}$ \\
\hline \multirow{4}{*}{ 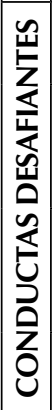 } & $\begin{array}{l}\text { Responder de forma rei- } \\
\text { terada }\end{array}$ & G & $\begin{array}{l}\text { Cambio de clase otro tutor/profesor. } \\
\text { Reflexión de las consecuencias. }\end{array}$ \\
\hline & $\begin{array}{l}\text { Desafiar al profesor/a y } \\
\text { agredir a un compañero/a }\end{array}$ & MG & $\begin{array}{l}\text { Expulsión de } 6 \text { días a un mes. } \\
\text { Seguimiento de trabajo de empatía, valores, emo- } \\
\text { ciones en la incorporación. }\end{array}$ \\
\hline & \begin{tabular}{|l|} 
Enfrentarse al profesor/a \\
\end{tabular} & MG & $\begin{array}{l}\text { Expulsión del recreo durante } 15 \text { días donde se reali- } \\
\text { zaran tareas para mejorar la conducta (se trabajará la } \\
\text { empatía, las consecuencias, figura del mediador). }\end{array}$ \\
\hline & $\begin{array}{l}\text { Conducta desafiante y } \\
\text { reiterada sanciones. }\end{array}$ & MG & $\begin{array}{l}\text { Expulsión de } 6 \text { días a un mes. } \\
\text { Mediación, respeto y consecuencias de los actos. }\end{array}$ \\
\hline
\end{tabular}

MG = Muy grave; $\mathrm{G}=$ Grave 
Además de las sanciones adoptadas en el centro, es importante que el alumnado y el profesorado participen en talleres para que se sensibilicen ante el problema de indisciplina y reconozcan sus causas, consecuencias y manifestaciones, y no naturalicen el problema. Así como, la asistencia a conferencias donde aprendan a no avalar las acciones disruptivas, la convivencia con valores, autoestima, empatía, aceptación de la diversidad, igualdad de género, entre otros. Conjuntamente, hacer un convenio de buena voluntad para crear un buen clima y ambiente de aprendizaje.

\section{Discusión}

Los hombres cometen más faltas (76\%), que las mujeres (24\%), estos datos coinciden con los reportados en diferentes investigaciones por Ruiz-Ramírez et al. (2017), Reyes et al. (2015) y Defensor del Pueblo (2007), quienes señalan que los hombres participan mayormente en actos de indisciplina o violencia escolar.

Con los resultados obtenidos se determina que en todos los grados escolares se comenten faltas de indisciplina, por lo que los niveles escolares no son limitantes para no ejercer actos de indisciplina, lo que coincide con Ruiz-Ramírez et al. (2016), Furlán (2009) y Berger et al. (2008).

Las faltas cometidas por los/as alumnos/as son reiteradas, en las edades tempranas, y están acompañadas por su falta de responsabilidad en la realización de sus tareas escolares. La mayoría de las faltas que se cometen son hacia el profesorado, esto coincide con Díaz-Aguado (2005) métodos rígidos de enseñanza.

La principal conducta disruptiva que se manifestó en esta investigación fue la agresión a compañeros/as, lo cual concuerda con las investigaciones realizadas por García (2010), Brunstein et al. (2007), Voors (2005) y Olweus (2006), quienes discutieron que las agresiones físicas son las que se presentan mayormente en actos de indisciplina.Lo que despierta una gran preocupación, para el ámbito escolar y los profesionales de la educación son las agresiones y amenazas que realizan, a temprana edad, los estudiantes en el entorno escolar. Como afirman autores como Olweus (2006) y Zapata-Martelo y Ruiz-Ramírez (2015) los problemas de acoso y amenazas entre escolares está aumentando, ya que en el ciclo escolar 2014-2015 fue de 24.5\% y en el ciclo escolar 2015-2016 aumentó a 26.5\%.

Por lo anterior, la aplicación de medidas disciplinarias hace que los motivos de las conductas disruptivas vayan bajando a lo largo de los cursos académicos siendo más altas en el curso académico del 2013 que en el 2016. En la línea de autores como Krskova y Baumann (2017) y Ruiz-Ramírez et al. (2018), quienes describen que los efectos de la indisciplina influyen el rendimiento escolar, con efectos negativos a lo largo del tiempo en los/as estudiantes.

Los factores a destacar en cuanto a la actitud del alumnado en cometer conductas disruptivas, alterando el clima de una convivencia en el centro escolar, según el análisis de estudio son los factores personales: Falta de inmadurez, incapacidad de controlar las situaciones con sus iguales, falta de autocontrol, rebeldía hacia el adulto, Ilamada de atención, lucha por el liderazgo dentro del grupo. Ante estos factores 
hay que tener en cuenta el perfil del alumnado y su entorno social (Ruiz-Ramírez et al., 2016; Ortega, 2008; Debarbieux y Blaya, 2006 y Reeve, 2006) para poder actuar y ayudarle a modificar su conducta para una convivencia compartida en el aula (Chang, 2004) y conseguir disminuir las faltas cometidas (Hoffman, 1994 y Preston, 1991).

Los datos evidencian la mejora en la evolución de los años escolares, la mejoría en el comportamiento es debida, a las medidas tomadas por el centro/s para mejorar la convivencia que parten de la prevención temprana, análisis de los factores, establecimiento de estrategias y el fomento de la participación de todos los agentes.

\section{Conclusiones}

Las actuaciones del alumnado de agresividad, insultos/amenazas y faltas de respeto hacia el profesorado, en el centro escolar, son actitudes cada vez más reiteradas que, se reflejan en el aula, desbordando en ocasiones la tarea docente. La aplicación de medidas disciplinarias desde edades tempranas hace que mejore el comportamiento de los estudiantes en cursos sucesivos. Por ello, establecer prácticas efectivas de disciplina es fundamental para garantizar el éxito académico y proporcionar un entorno de aprendizaje seguro (Luiselli, Putnam, Handler, y Feinberg, 2005)

Al encontrarnos ante un problema real que cada vez más surgen en las aulas en las etapas tempranas donde el papel del profesorado, familia es primordial y fundamental. La percepción del profesorado en la gravedad de las faltas cometidas es muy importante, la finalidad es que se ha determinar la sanción a dichas faltas y poner las medidas necesarias de apoyo (dialogo, mediación entre iguales...) para que estas no se vuelvan a producir y realizar un seguimiento de las mismas. En la línea de autores como (Gregory et al., 2010) sobre la necesidad del cumplimiento consistente de la disciplina escolar (estructura) y la disponibilidad de adultos comprensivos (apoyo) que se asocian con la seguridad de la escuela. Sus hallazgos sugieren que las prácticas disciplinarias no deben polarizarse en un debate de "ponerse duro" frente a "dar apoyo" porque tanto la estructura como el apoyo contribuyen a la seguridad escolar de los estudiantes.

El papel de la familia es fundamental y esencial, aunque a veces es difícil contar con su participación, por tanto, en multitud de casos las medidas de corrección recaen sobre las medidas que toma en centro, en mejorar dicha convivencia.

En línea de Tobin y Sugai (1996) desarrollar en un centro registro de disciplina permite establecer predictores de comportamientos violentos. El uso de patrones de referencia de disciplina escolar como un medio para verificar la necesidad de evaluaciones adicionales de la conducta en los estudiantes para prevenir dichos comportamientos.

Ante esta problemática se debe reflexionar y pensar que se requiere actuar desde los centros y la familia de forma urgente, para poder prevenir que se desencadenen conflictos en los centros educativos, subsanar los que se han desencadenado y realizar un seguimiento para que no afloren comportamientos y conflictos que alteren la vida escolar. 


\section{ReferenCias BibLIOGRáficas}

Berger, C., Karimpour, R. y Rodkin, P. (2008). Bullies and victims at school: Perspectives and strategies for primary prevention. En: Tomas W. Miller (Ed.), School violence and primary prevention. New York, Springer, pp. 295-322.

Brophy-Herb, H. E., Lee, R. E., Nievar, M. A. y Stollak, G. (2007). Preschoolers' social competence: Relations to family characteristics, teacher behaviors and classroom climate. Journal of Applied Developmental Psychology, 28(2), 134-148. https:// doi.org/10.1016/j.appdev.2006.12.004.

Brunstein, A., Marrocco, F., Kleiman, M., Schonfeld, I. y Gould, M. (2007). Bullying, depression, and suicidality in adolescents. J Am Acad Child Adolesc Psychiatry, 46(1), 40-49.

Chang, L. (2004). The Role of Classroom Norms in Contextualizing the Relations of Children's Social Behaviors to Peer Acceptance. Developmental Psychology, 40(5), 691-702. https://doi.org/10.1037/0012-1649.40.5.691.

Chen, J.-K. y Avi Astor, R. (2010). SchoolViolence in Taiwan: Examining How Western Risk Factors Predict School Violence in an Asian Culture. Journal of Interpersonal Violence, 25(8), 1388-1410. https://doi.org/10.1177/0886260509354576.

Creswell, J. W. L. y Clark, V. P. (2010). Choosing a mixed methods design. En Designing and conducting mixed methods research (pp. 53-106). https://doi. org/1412927927.

Debarbieux, E. y Catherine, B. (2006). Clima y violencia escolar: Un estudio comparativo entre España y Francia. Revista de Educación, 339, 293-315.

Defensor del Pueblo.(2007), Violencia escolar: El maltrato entre iguales en la educación secundaria obligatoria 1999-2006. Madrid, Comité Español de UNICEF, Publicaciones de la Oficina del Defensor del Pueblo.

Del Tronco, J. (2013), La violencia en las escuelas secundarias de México. Una exploración de sus dimensiones. México, FLACSO.

Denzin, N. K. (2010). Moments, Mixed Methods, and Paradigm Dialogs. Qualitative Inquiry, 16(6), 419-427. https://doi.org/10.1177/1077800410364608.

Díaz-Aguado Jalón, M. J. (2005). La violencia entre iguales en la adolescencia y su prevención desde la Escuela. Psicothema, 17(4), 449-558.

Evans, C. B. R., Fraser, M. W. y Cotter, K. L. (2014). The effectiveness of schoolbased bullying prevention programs: A systematic review. Aggression and Violent Behavior, 19(5), 532-544. https://doi.org/10.1016/j.avb.2014.07.004.

Farrell, A. D., Meyer, A. L. y White, K. S. (2001). Evaluation of Responding in Peaceful and Positive Ways (RIPP): A School-Based Prevention Program for Reducing Violence Among Urban Adolescents. Journal of Clinical Child y Adolescent Psychology, 30(4), 451-463. https://doi.org/10.1207/ S15374424JCCP3004_02. 
Flannery, D. J., Vazsonyi, A. T., Liau, A. K., Guo, S., Powell, K. E., Atha, H., ... Embry, D. (2003). Initial behavior outcomes for the PeaceBuilders universal school-based violence prevention program. Developmental Psychology, 39(2), 292-308. https:// doi.org/10.1037/0012-1649.39.2.292.

Foshee, V. A., Benefield, T. S., McNaughton Reyes, H. L., Eastman, M., Vivolo-Kantor, A. M., Basile, K. C., ... Faris, R. (2016). Examining explanations for the link between bullying perpetration and physical dating violence perpetration: Do they vary by bullying victimization? Aggressive Behavior, 42(1), 66-81. https://doi.org/10.1002/ab.21606.

Furlán, A. (2009). Acerca de la violencia en la escuela. Revista Novedades Educativas, 224 , pp. 12-15.

Galand, B., Lecocq, C. y Philippot, P. (2007). School violence and teacher professional disengagement. British Journal of Educational Psychology, 77(2), 465-477. https://doi.org/10.1348/000709906X114571.

Garaigordobil, M. y Oñederra, J. (2010). Inteligencia emocional en las víctimas de acoso escolar y en los agresores. European Journal of Education and Psychology, 3(2), 243-256. https://doi.org/10.1989/ejep.v3i2.63.

Garaigordobil Landazabal, M. y Oñederra, J. A. (2010). Los centros educativos ante el acoso escolar: actuaciones del profesorado, acciones sancionadoras y actividades de acciones sancionadoras y actividades de prevención. Informació Psicològica, 99, 4-18. https://doi.org/10.1017/CBO9781107415324.004.

García-Costoya, M. (2010). Violencia en las escuelas. Un relevamiento desde la mirada de los alumnos. Buenos Aires: Ministerio de Educación.

Gregory, A., Cornell, D., Fan, X., Sheras, P., Shih, T.-H. y Huang, F. (2010). Authoritative school discipline: High school practices associated with lower bullying and victimization. Journal of Educational Psychology, 102(2), 483-496. https://doi. org/10.1037/a0018562.

Hoffman, M. L. (1994). Discipline and internalization. Developmental Psychology, 30(1), 26-28. https://doi.org/10.1037//0012-1649.30.1.26.

Hunter, L., Elias, M. J. y Norris, J. (2001). School-based violence prevention. Journal of School Psychology, 39(2), 161-175. https://doi.org/10.1016/S00224405(01)00058-9.

Idu, A. P. y Ojedapo, D. O. (2011). Indiscipline in Secondary Schools: A Cry to All Stakeholders in Education. Proceedings of the 2011 International Conference on Teaching, Learning and Change, (c), 729-735.

Johnson, B., Onwuegbuzie, A. J. y Turner, L. A. (2007). Toward a Definition of Mixed Methods Research. Journal of Mixed Methods Research, 1(2), 112-133. https://doi. org/10.1177/1558689806298224.

Johnson, S. L. (2009). Improving the school environment to reduce school violence: A review of the literature. Journal of School Health, 79(10), 451-465. https://doi. org/10.1111/j.1746-1561.2009.00435.x. 
Kinsler, J. (2013). School discipline: A source or salve for the racial achievement gap? International Economic Review, 54(1), 355-383. https://doi.org/10.1111/j.14682354.2012.00736.x.

Krskova, H. y Baumann, C. (2017). School discipline, investment, competitiveness and mediating educational performance. International Journal of Educational Management, 31, 293-319. https://doi.org/10.1108/IJEM-05-2016-0099.

Luengo, F. (2006). El Proyecto Atlántida : experiencias para fortalecer el eje escuela, familia y municipio. Revista de Educación, 339, 177-193.

Luiselli, J. K., Putnam, R. F., Handler, M. W. y Feinberg, A. B. (2005). Whole-school positive behaviour support: Effects on student discipline problems and academic performance. Educational Psychology. https://doi.org/10.1080/0144341042000301265.

Maphosa, C. y Mammen, K. J. (2011). How chaotic and unmanageable classrooms have become: Insights into prevalent forms of learner indiscipline in south african schools. Anthropologist, 13(3), 185-193.

Marcus, R. F. (2007). Aggression and Violence in Adolescence. Cambridge: Cambridge University Press. https://doi.org/10.1017/CBO9780511611292.

McKinney, C. M., Caetano, R., Ramisetty-Mikler, S. y Nelson, S. (2009). Childhood Family Violence and Perpetration and Victimization of Intimate Partner Violence: Findings From a National Population-Based Study of Couples. Annals of Epidemiology, 19(1), 25-32. https://doi.org/10.1016/j.annepidem.2008.08.008.

Meirieu, P. (2001). La Opción de educar :ética y pedagogía. Barcelona: Octaedro.

Olweus, D. (2001). Bullying at school: Tackling the problem. OECD Observer, 225, 24-26. https://doi.org/10.1007/978-1-4757-9116-7_5.

Olweus, D. (2006). Conductas de acoso y amenaza entre escolares. Madrid: Morata.

Ortega-Rivera, J., Sánchez-Jiménez, V. y Ortega-Ruiz, R. (2010). Violencia sexual y cortejo juvenil. En Rosario Ortega-Ruiz (Coord.), Agresividad injustificada, buIlying y violencia escolar. Madrid: Alianza Editorial, pp. 211-232.

Peter A. Hastie, y Siedentop, D. (2006). The classroom ecology paradigm. En The handbook of physical education (p. 838). https://doi.org/10.4135/9781848608009.

Peterson, R. L. y Skiba, R. (2001). Creating School Climates That Prevent School Violence. Clearing House, 74(3), 155-163. https://doi. org/10.1080/10459880009599794.

Piñuel, I. y Oñate, A. (2006). La evaluación y diagnóstico del mobbing o acoso psicológico en la organización: el barómetro Cisneros. Revista de Psicología del Trabajo y de las Organizaciones = Journal of Work and Organizational Psychology, 22(3), 309-332. Recuperado de http://dialnet.unirioja.es/servlet/articulo?codigo=22297 01 yinfo=resumenyidioma $=$ SPA

Preston, P. (1991). Positive discipline. Administrative Radiology : AR, 10(7), 17-20. https://doi.org/ISBN: 978-974-7522-68-6. 
Reeve, J. (2006). Teachers as Facilitators: What Autonomy-Supportive Teachers do and Why their Students Benefit. The Elementary School Journal, 106(3), 225-236.

Reyes-Sánchez, C., Martínez-Ferrer, B. y Moreno, D. (2015). Adolescentes agresores en la escuela. Un análisis desde la perspectiva de género. Feminismo/s, 25, 111-131.

Rimm-Kaufman, S. E., Curby, T. W., Grimm, K. J., Nathanson, L. y Brock, L. L. (2009). The contribution of children's self-regulation and classroom quality to children's adaptive behaviors in the kindergarten classroom. Developmental Psychology, 45(4), 958-972. https://doi.org/10.1037/a0015861.

Ruíz-Ramírez, R., Sánchez Romero, C., Zapata-Martelo, E., García-Cué, J. L., PérezOlvera, M. A., Martínez-Corona, B. y Rojo-Martínez, G. (2017). Manifestaciones del bullying en la Preparatoria Agrícola. Universidad Autónoma de Chapingo, México. Revista Latinoamericana de Ciencias Sociales, Niñez y Juventud, 15(2), 1149-1163.

Ruiz-Ramírez, R., Zapata-Martelo, E., García-Cué, J. L., Pérez-Olvera, A., MartínezCorona, B. y Rojo-Martínez, G. (2016). Bullying en una Universidad Agrícola del Estado de México. Ra Ximhai, 12(1), 105-126.

Ruíz-Ramirez, R., García-Cué, J. L., Ruíz-Martínez, F. y Ruíz-Martínez, A. (2018). La relación bullying-deserción escolar en bachilleratos rurales. Revista Electrónica de Investigación Educativa, 20(2), 37-45.

Schultes, M. T., Stefanek, E., van de Schoot, R., Strohmeier, D. y Spiel, C. (2014). Measuring implementation of a school-based violence prevention program: Fidelity and teachers' responsiveness as predictors of proximal outcomes. Zeitschrift Fur Psychologie / Journal of Psychology, 222(1), 49-57. https://doi.org/10.1027/21512604/a000165.

Shapiro, J. P., Burgoon, J. D., Welker, C. J. y Clough, J. B. (2002). Evaluation of the Peacemakers Program: School-based violence prevention for students in grades four through eight. Psychology in the Schools, 39(1), 87-100. https://doi. org/10.1002/pits.10040.

Simon, T. R., Ikeda, R. M., Smith, E. P., Reese, L. E., Rabiner, D. L., Miller-Johnson, S., ... Allison, K. W. (2008). The multisite violence prevention project: Impact of a universal school-based violence prevention program on social-cognitive outcomes. Prevention Science, 9(4), 231-244. https://doi.org/10.1007/s11121-008-0101-1.

Tashakkori, A. y Creswell, J. W. (2007). Exploring the nature of research questions in mixed methods research. Journal of Mixed Methods Research. https://doi. org/10.1177/1558689807302814.

Teddlie, C. y Tashakkori, A. (2010). Overview of contemporary issues in mixed methods research. Sage Handbook of Mixed Methods in Social y Behavioral Research, 1-44. https://doi.org/10.4135/9781506335193.n1.

Teddlie, C. y Yu, F. (2007). Mixed Methods Sampling: A Typology with Examples. Journal of Mixed Methods Research, 1(1), 77-77. https://doi. org/10.1177/1558689806292430. 
Terrell, S. R. (2012). Mixed-Methods Research Methodologies. The Qualitative Report, 17(1), 254-280. https://doi.org/10.1177/1744987106064635.

The Anti-Bullying Alliance. (2009). Bullying. Children. Recuperado de http://www. anti-bullyingalliance.org.uk/pdf/bullying_NCB_LIS_highlight261.pdf.

Tobin, T. y Sugai, G. (1996). Patterns in middle school discipline records. Journal of Emotional y Behavioral Disorders, 4(2), 82-95. https://doi. org/10.1177/106342669600400203.

Torrego Seijo, J. C. (Coord.) (2006). Modelo integrado de mejora de la convivencia: estrategias de mediación y tratamiento de conflictos. Barcelona: Grao.

Van der Horst, C. y Narodowski, M. (1999). Orden y disciplina. Educação y Realidade, 24.

Van Goethem, A. A. J., Scholte, R. H. J. y Wiers, R. W. (2010). Explicit-and implicit bullying attitudes in relation to bullying behavior. Journal of Abnormal Child Psychology, 38(6), 829-842. https://doi.org/10.1007/s10802-010-9405-2.

Watson, C. (2005). Discourses of “indiscipline": a Foucauldian response. Emotional and BehaviouralDifficulties, 10(1), 55-65.https://doi.org/10.1080/1363275205052530.

Voors, W. (2005). Bullying el Acoso Escolar. Barcelona: Oniro.

Yoon, J. S. y Kerber, K. (2003). Bullying. Research in Education, 27. Recuperado de http://search.ebscohost.com/login.aspx?direct=trueydb=ehhyAN=9810081ysite= ehost-live.

Zapata-Martelo, E. y Ruiz-Ramírez, R. (2015). Respuestas institucionales ante la violencia escolar. Ra Ximhai, 11(4), 475-491. 